\title{
The impact of physiological noise on hemodynamic-derived estimates of directed functional connectivity
}

F. Konrad Schumacher ${ }^{1,2,3,4,5}$, Carmen Steinborn ${ }^{1,2,3,4}$, Cornelius Weiller ${ }^{1,2,4,5}$, Björn O. Schelter ${ }^{5,6}$, Matthias Reinhard ${ }^{4,7}$, Christoph P. Kaller ${ }^{1,2,4,5,8}$

${ }^{1}$ Dept. of Neurology, Medical Center - University of Freiburg, 79106 Freiburg, Germany $\mid{ }^{2}$ Freiburg Brain Imaging Center, University of Freiburg, 79106 Freiburg, Germany $\left.\right|^{3}$ Faculty of Biology, University of Freiburg, 79104 Freiburg, Germany $\mid{ }^{4}$ Faculty of Medicine, University of Freiburg, 79085 Freiburg, Germany $\mid{ }^{5}$ BrainLinks-BrainTools Cluster of Excellence, University of Freiburg, 79110 Freiburg, Germany $\mid{ }^{6}$ Institute for Complex Systems and Mathematical Biology, University of Aberdeen, Aberdeen AB24 3UE, UK $\mid{ }^{7}$ Department of Neurology, Medical Center Esslingen, Teaching Hospital of the University of Tübingen, Esslingen, Germany $\mid{ }^{8}$ Department of Neuroradiology, Medical Center - University of Freiburg, 79106 Freiburg, Germany

Running title: The impact of noise on functional connectivity

Acknowledgement: This work was supported by a grant of the BrainLinks-BrainTools Cluster of Excellence funded by the German Research Foundation (DFG, grant number EXC 1086).

Corresponding author: Dr. Christoph Kaller (christoph.kaller@uniklinik-freiburg.de)

Dept. of Neuroradiology, Medical Center - University of Freiburg, Breisacher Str. 64, 79106 Freiburg, Germany 


\section{Abstract}

2 Measuring the strength of directed functional interactions between brain regions is fundamental to 3 understand neural networks. Functional near-infrared spectroscopy (fNIRS) is a suitable method to 4 map directed interactions between brain regions but is based on the neurovascular coupling. It thus relies on vasomotor reactivity and is potentially biased by non-neural physiological noise. To investigate the impact of physiological noise on fNIRS-based estimates of directed functional connectivity within the rostro-caudal hierarchical organization of the prefrontal cortex (PFC), we systematically assessed the effects pathological perturbations of vasomotor reactivity and externally triggered arterial blood pressure (aBP) fluctuations.

Fifteen patients with unilateral stenosis of the internal carotid artery (ICA) underwent multi-channel fNIRS during rest and during metronomic breathing, inducing aBP oscillations at .1 Hz. Comparisons between the healthy and pathological hemispheres served as quasi-experimental manipulation of the neurovascular system's capability for vasomotor reactivity. Comparisons between rest and breathing served as experimental manipulation of two different levels of physiological noise that were expected to differ between healthy and pathological hemispheres.

In the hemisphere affected by ICA stenosis, the rostro-caudal hierarchical organization of the PFC was compromised reflecting the pathological effect on the vascular and neural level. Breathing-induced aBP oscillations biased the magnitude of directed interactions in the PFC, but could be adjusted using either the aBP time series (intra-individual approach) or the aBP-induced fNIRS signal variance (interindividual approach). Multi-channel fNIRS hence provides a sound basis for analyses of directed functional connectivity as potential bias due to physiological noise can be effectively controlled for.

\section{Key words}

Prefrontal cortex; Hierarchical organization; Directed interactions; Near-infrared spectroscopy; Physiological noise; Stenosis 


\section{Abbreviations}

arterial blood pressure (aBP); functional magnetic resonance imaging (fMRI); functional near-infrared spectroscopy (fNIRS); prefrontal cortex (PFC); internal carotid artery (ICA); (partial) directed coherence ((P)DC); power spectral density (PSD); vector autoregressive (VAR)

\section{Compliance with Ethical Standards}

Conflicts of interest. The authors declare that they have no conflict of interest.

Funding. This work was supported by a grant of the BrainLinks-BrainTools Cluster of Excellence funded by the German Research Foundation (DFG, grant number EXC 1086).

Ethical approval. The study was approved by the local Ethics Committee.

Informed consent. All patients gave written informed consent prior to participation 


\section{Introduction}

Understanding functional networks of the brain is an ongoing challenge in human neuroscience. Approaches to disentangle the functional dynamics between regions of the human brain are mainly based on non-invasive imaging methods that often face tradeoffs between signal-to-noise ratio, temporal and spatial resolution (Scouten et al. 2006). Given the high spatial but low temporal resolution of the commonly used functional magnetic resonance imaging (fMRI), most extant approaches on connectivity are based on correlative measures. However, a high temporal resolution is particularly critical when the directionalities of functional connections are of interest (Roebroeck et al. 2005; Mader et al. 2008). Multi-channel functional near-infrared spectroscopy (fNIRS) as an optical method to measure cortical hemodynamics provides such high temporal resolutions, an adequate signal quality and a sufficient spatial resolution to assess large-scale cortical networks.

Schumacher et al. (2019) recently demonstrated that Granger-causal cross-spectral analysis (Granger 1969; Schelter et al. 2006) of resting-state fNIRS data is a promising approach to characterize the rostro-caudally directed hierarchical organization of the prefrontal cortex (PFC; see also Medvedev, 2014). The functional architecture of the PFC is thought to implement different levels of cognitive control by processing information through a rostral-to-caudal hierarchy of neural networks, thereby concretizing abstract ideas into actual actions according to specific rules (Badre and D'Esposito, 2007; Blumenfeld et al., 2013; Christoff and Gabrieli, 2000; Fuster, 2008; Koechlin et al., 2003; for a recent review see Badre and Nee, 2018). Directly assessing the mode of action of the PFC and its integrity with a convenient, high-resolution imaging method like multi-channel fNIRS may hence constitute a promising approach for cognitive and clinical neuroscience that complements insights derived from conventional fMRI.

However, the suitability of Granger-causality for analyses of hemodynamic measurements was subject to controversy in the past (Friston et al., 2014; Schippers et al., 2011; Smith et al., 2012; Stokes and Purdon, 2017; Webb et al., 2013; for reviews see Deshpande and Hu, 2012; Friston et al., 2013). Specifically, the sampling rate of the measurement relative to the time scale of the causal mechanism has been identified as a critical parameter (Deshpande et al. 2010; Barnett and Seth 2017) - an issue 
already put forward by Granger (1969). Yet, while this constitutes a serious limitation for fMRI with sampling rates of only $.5-2 \mathrm{~Hz}$, fNIRS samples at an order of magnitude faster. Commercially available multi-channel fNIRS systems usually have sampling rates in the range of $10-250 \mathrm{~Hz}$ (Scholkmann et al. 2014) thus providing a sufficient temporal resolution of at least $10 \mathrm{~Hz}$ (Roebroeck et al. 2005) for estimating the directionality of influences within large-scale cortical networks.

The impact of physiological noise on Granger-causality inference is another potential issue of concern which has previously received only little attention. Physiological noise particularly concerns analyses of fNIRS data as the near-infrared light has to traverse the scalp and the skull before reaching the brain and thus also samples from extra-cerebral (i.e. non-neural) tissue (Okada et al. 1997; Germon et al. 1999; Brigadoi and Cooper 2015). Apart from the extra-cerebral signal component, spontaneous slow oscillations in the arterial blood pressure (aBP) induce autoregulatory vasomotor activity (Julien 2006) and contribute to intra-cerebral signal variance in the frequency band of .1 $\mathrm{Hz}$ (Tong and Frederick 2010; Noordmans et al. 2018) commonly used for connectivity analyses (Biswal et al. 1995). Besides these aBP-induced low frequency oscillations other systemic components originating from cardiac pulsation, respiration and vasomotion unrelated to neural activity can bias analyses based on functional measurements of brain hemodynamics (Frederick et al. 2012; Winder et al. 2017). These components not only differ in their spectral properties, but also in their propagation along the vasculature, i.e. they exhibit different spatiotemporal profiles (Frederick et al. 2012; Tong et al. 2012). Optical measurements of blood oxygenation at the periphery (e.g. the finger) provide an easy way to capture a wide spectrum of systemic hemodynamic processes and can be used to reduce physiological noise in functional measurements (Frederick et al. 2012; Tong et al. 2013; Sutoko et al. 2019).

Analyses contrasting different conditions in a task paradigm to find cortical activations associated with specific brain functions are generally assumed to be robust against extra-cerebral physiological noise as well as against intra-cerebral aBP fluctuations (but see Takahashi et al., 2011). However, such signal perturbations may possibly limit the reliability and validity of fNIRS-based Granger-causal cross-spectral estimates of directed connectivity. More generally, any estimation of (directed as well as undirected) connectivity based on hemodynamic neuroimaging such as fNIRS (but also fMRI) faces 
the problem of distinguishing signal covariation induced by neural activity from those induced by nonneural fluctuations of blood flow, blood pressure, or respiration (Tong et al. 2013; Pfurtscheller et al. 2017). Concerning resting-state functional connectivity estimated from hemodynamic measurements, it is hence critical to minimize the impact of physiological noise and to ascertain that the measurement is sensitive and specific to neural processes.

The present study therefore addressed the effects of physiological noise and pathological cerebral hemodynamics on Granger-causal cross-spectral analyses of directed connectivity based on multichannel fNIRS data. Specifically, we investigated the influence of peripherally induced physiological noise and impaired vasomotor reactivity on the reconstruction of the rostro-caudally directed hierarchical organization in the PFC using a frequency-domain measure of Granger-causality (cf. Schumacher et al., 2019). To this end, a sample of patients with unilateral stenosis of the internal carotid artery (ICA) was assessed with fNIRS during metronomic breathing and during rest. ICA stenosis leads to reduced cerebrovascular reserve capacity (Bokkers et al. 2010; Hartkamp et al. 2012), impairment of cerebral autoregulation (Reinhard et al. 2003b), affects neurovascular coupling (Rossini et al. 2004) and can cause cognitive impairments (Novak and Hajjar 2010; Novak 2012). As severe ICA stenosis impairs cerebral autoregulation (Reinhard et al. 2003b), the hemisphere affected by ICA stenosis has only limited capacity to compensate systemic low-frequency high-amplitude aBP oscillations. Metronomic breathing induces strong peripheral physiological noise in terms of lowfrequency arterial blood pressure (aBP) oscillations that contaminate the fNIRS signal. Comparing resting-state and metronomic breathing in patients with unilateral ICA stenosis thus allows differentiating between the systemic effect of amplified peripheral physiological noise (i.e. aBP oscillations), which contributes to both the intra- and extra-cerebral components of the fNIRS signal, and the intra-cerebral effect of a compromised neurovascular system (i.e. ICA stenosis), that was expected to cause differential effects of breathing-induced aBP oscillations in the patients' healthy and pathological hemispheres. 


\section{Methods}

\section{Patients}

118 Fifteen patients with severe unilateral stenosis or occlusion of the internal carotid artery (ICA) were

119 included and gave written informed consent prior to participation (also see Reinhard et al., 2014). The advantage of using unilateral stenosis of the ICA as a quasi-experimental manipulation is that it facilitates the assessment of pathological effects by comparing the affected and healthy hemisphere within patients without relying on a healthy control group, yielding a higher statistical power. Transcranial duplex sonography was used to determine the degree of stenosis (de Bray and Glatt 1995) and the degree of intracranial collateral flow (Reinhard et al. 2003a). Magnetic resonance imaging (MRI) scans were acquired from all patients and revealed a large lesion from the resection of a hemangioblastoma in one patient, who was consequently excluded from the present analyses. A second patient had to be excluded due to technical problems during the resting-state fNIRS measurement, leaving a sample of 13 patients for analyses (mean age \pm standard deviation: $63.5 \pm 10$ years, 3 female; see Reinhard et al., 2014 for further details). The study was approved by the local

Ethics Committee.

\section{Data acquisition}

132 Arterial blood pressure (aBP) was continuously recorded via finger photoplethysmography (Finapres 2300, Ohmeda, Englewood, CO, USA) with the subject's hand positioned at heart level. Multi-channel fNIRS measurements were performed using an ETG 4000 (Hitachi Medical Co., Tokyo, Japan) providing 52 channels and a sampling rate of $10 \mathrm{~Hz}$. In-house Matlab (version 2015a, The MathWorks, Natick, MA, USA) code was used to convert the measured light absorption into oxygenated and deoxygenated hemoglobin concentration changes according to the modified LambertBeer-Law (Delpy et al. 1988). The fNIRS probes were placed on the forehead by aligning the center probes with the sagittal midline and positioning the lower center probe at a distance of $1.5 \mathrm{~cm}$ above the nasion, such that the fNIRS channels were evenly distributed across the bilateral PFC. The fNIRS channel positions are illustrated in Figure 1. Patients were placed in a supine position with $50^{\circ}$ inclination of the upper body. The first measurement was conducted during a 15 minute period of rest. 
143 During the second measurement patients were instructed to breath at a rate of 6 cycles/minute (i.e. .1

$144 \mathrm{~Hz}$ ) with low tidal volumes over a period of $200 \mathrm{~s} . \mathrm{CO}_{2}$ partial pressure was measured during 145 expiration using an infrared capnometer (Normocap $\odot$, Datex, Finland). We analyzed only the first 200

$146 \mathrm{~s}$ of the resting state measurement, because the length of the time series potentially impacts on the connectivity estimation (see below) and the direct comparison between 15 min resting state and $200 \mathrm{~s}$ metronomic breathing would have hence been biased. However, a control analysis confirmed that there were no relevant differences between 4 consecutive, $200 \mathrm{~s}$ long time windows of the $15 \mathrm{~min}$ resting-state measurements (Supplementary Model S1).

\section{Data analysis}

152 The aBP signal was low-pass filtered (Fourier filter, $5 \mathrm{~Hz}$ cutoff frequency) and downsampled to the $10 \mathrm{~Hz}$ sampling rate of the fNIRS measurement. To avoid bias of the Granger-causality estimates (see below), the fNIRS data was not filtered or resampled (Florin et al. 2010; Barnett and Seth 2011). However, as fNIRS is prone to movement-induced artifacts, which can cause spurious connectivity (Satterthwaite et al. 2012; Santosa et al. 2017), the preprocessing requires an artifact correction step. Therefore, we applied the correlation-based signal improvement (CBSI) (Cui et al. 2010), an established method that effectively removes motion artifacts, increases the contrast-to-noise ratio and enhances the sensitivity of the signal (Cui et al. 2010; Brigadoi et al. 2014; Racz et al. 2017; Fairclough et al. 2018). It is based on the assumption that the oxygenated and the deoxygenated hemoglobin concentrations are anticorrelated; as a consequence, the resulting time series of oxygenated and deoxygenated hemoglobin are perfectly anticorrelated and have identical spectral properties. However, the assumptions implied by the CBSI method are unlikely to be always met. We therefore provide supplementary control analyses of the connectivity derived from the uncorrected oxygenated and deoxygenated hemoglobin signals (Supplementary Models S2-S5). Directed

166 functional connectivity was estimated from the fNIRS measurements by directed coherence (DC)

167 (Schelter et al. 2006), a cross-spectral measure of Granger-causality, using the frequency domain multivariate toolbox (www.fdm.uni-freiburg.de/Toolboxes/fdma-toolbox). As indicated by the term coherence, DC is a frequency-domain measure and is calculated by fitting a vector autoregressive

170 (VAR) model, i.e. each time series is explained by its own past, as well as by the past of at least one 
second time series using multiple lags. The series of estimated autoregression coefficients resulting from the multiple lags are then transformed into the frequency domain (for details see Schelter et al., 2006). Thus, DC estimated from fNIRS data represents the strength and the direction of influences exerted between cortical areas in a certain frequency. For the connectivity estimates corrected for the potential bias of aPB fluctuations, we included the aBP measurements in the VAR models, yielding the trivariate - i.e. partial - DC (PDC). Thus, PDC estimates represent the influences between cortical areas with the influences mediated by systemic physiological noise (i.e. aBP fluctuations) being removed. VAR models were fitted with a model order of 20 (i.e. 20 lags), corresponding to the past $2 \mathrm{~s}$ of the time series., The resulting model coefficients were zero-padded to the length of the time series before the Fourier transformation in order to yield a smooth spectral estimate. As functional connectivity is apparent in the low-frequency component of hemodynamic fluctuations (Biswal et al. 1995), and to cover the power peak in the fNIRS signal induced by metronomic breathing at .1 Hz, we used the maximum between .06 and $.12 \mathrm{~Hz}$ of each (P)DC spectrum for further statistical analysis. The (P)DC was entered as the dependent variable in linear mixed effects models. Mixed models were fitted using the lme4 package (version 1.1-14) (Bates et al. 2015) in R statistics (version 3.4.2; http://cran.rproject.org) with unstructured variance-covariance matrices. The lmerTest package (version 2.0-33) (Kuznetsova et al. 2016) was used to assess the significance of predictor terms (Type III F-statistics with Satterthwaite's approximation of degrees of freedom). Post-hoc comparisons and calculation of confidence intervals were performed using the lsmeans package (version 2.27-2) (Lenth 2016). As the focus of the present study was on the rostro-caudal gradient of the PFC, fixed effects of all models contained the factor direction of influences, distinguishing between influences in rostral-to-caudal and caudal-to-rostral direction (Fig. 1). As recently shown (Schumacher et al. 2019), the directionality of rostro-caudal functional connectivity varies across PFC regions (see also Badre and Nee, 2018; Margulies et al., 2016; Nee and D’Esposito, 2016). In accordance, present models were specified with a random slope allowing for varying effects of direction for each pair of homologues connections.

196 Additionally a random intercept for participant was included in each model. Fixed effect terms of each

197 model are listed in Table 1; predicted marginal means are provided in Supplementary Table 1; for random effects see Supplementary Table 2. 


\section{Data visualization}

200

201

202

203

204

205

F1
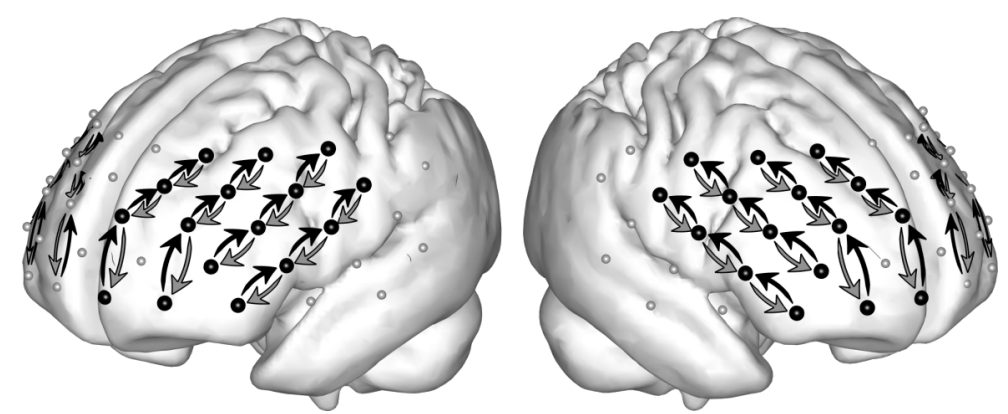

214 Figure 1: Connections between fNIRS channels analyzed in the present study. Directed connectivity between

215 neighboring channels within the PFC was analyzed along the rostro-caudal axis. Black arrows indicate rostrocaudally directed connections, whereas gray arrows indicate caudo-rostrally directed connections.

\section{Results}

218 In the present work we analyzed the effects of amplified peripheral physiological noise (i.e. aBP oscillations) and of a compromised vasomotor reactivity (i.e. ICA stenosis) on the estimation of the rostro-caudally directed hierarchical organization of the PFC. Multi-channel fNIRS measurements 
were acquired in patients with unilateral ICA stenosis during resting state and during metronomic breathing at .1 Hz. Directed functional connectivity was analyzed as the dependent variable in linear mixed models with a random slope allowing for varying effects of direction (distinguishing between influences in rostral-to-caudal and caudal-to-rostral direction) for each pair of homologues connections (cf. Fig. 1). Additionally a random intercept for participant was included in each model. The fixed effects structure is described for each model in the following. An overview of the applied statistical models is provided in Table 1. The predicted marginal means and corresponding confidence intervals for significant effects are provided in Supplementary Table S1.

\section{Metronomic breathing increases blood pressure oscillations and overall connectivity strength}

As manipulation check, a one-sample t-test comparing the peak power spectral density (PSD) of the continuous aBP measurement in the frequency band between .06 and $.12 \mathrm{~Hz}$ confirmed that the metronomic breathing induced strong aBP oscillations compared to the resting state (mean difference: $6.1 \mathrm{~dB} ; \mathrm{t}(12)=5.1 ; \mathrm{p}=.0003$; Fig. 2, also see Fig. 5 for fNIRS and aBP PSD spectra).

$F 2$

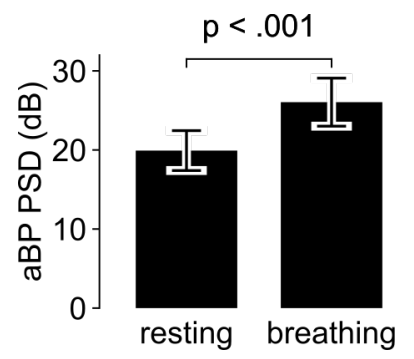

Figure 2: The low-frequency power spectral density (PSD) of the arterial blood pressure (aBP) was strongly increased during metronomic breathing compared to resting state. Bars represent group means; error bars indicate $95 \%$ confidence intervals; $\mathrm{p}$-value refers to a one-sample two-tailed t-test.

The first analysis of directed functional connectivity addressed the hypothesis that the impaired cerebral vasomotor reactvity in the hemisphere affected by ICA stenosis would specifically attenuate the rostro-caudal gradient, while the functional gradient was expected to be generally robust against increased aBP oscillations induced by metronomic breathing. To this end, we fitted a linear mixed model (Model 1) with a fixed effects structure comprising the three-way interaction (and all main

243 effects and lower-order interactions) between direction (rostro-caudal vs. caudo-rostral), hemisphere 
244 (affected vs. healthy) and condition (resting state vs. metronomic breathing). The main effect for 245 direction confirmed the predominance of rostro-caudally directed influences $(F(1,12)=14.6, p=.002)$

246 with higher DC estimates between adjacent fNIRS channels in rostro-caudal than in caudo-rostral

247 direction. A strong main effect for condition $(\mathrm{F}(1,1212)=61.0, \mathrm{p}<.0001)$ indicated that deep

248 breathing led to an overall increase in connectivity strength. The two-way interaction between

249 direction and hemisphere $(\mathrm{F}(1,1212)=12.2, \mathrm{p}=.0005)$ further indicated that ICA stenosis attenuates

250 the rostro-caudal gradient. However, the significant three-way interaction between direction, 251 hemisphere, and condition $(\mathrm{F}(1,1212)=4.5, \mathrm{p}=.034)$ revealed that the impact of breathing-induced 252 aBP oscillations on the rostro-caudal gradient was different between hemispheres (Fig. 3): The 253 difference between the directions of influences in the healthy hemisphere was larger during 254 metronomic breathing than during rest $(\mathrm{p}=.040)$ while there was no significant difference in 255 directionality between conditions in the affected hemisphere $(\mathrm{p}=.349)$. Neither the main effect for 256 hemisphere $(\mathrm{p}=.642)$, nor the other two-way interactions were significant (all $\mathrm{p}>.424)$. Taken 257 together, the rostro-caudal gradient in the PFC was significantly increased in the healthy hemisphere 258 by metronomic breathing while it was stable in the hemisphere affected by ICA stenosis. 
A

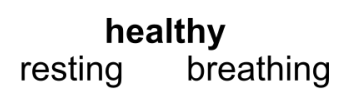

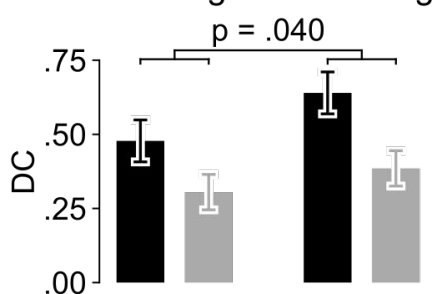

rostral $\rightarrow$ caudal caudal $\rightarrow$ rostral

\section{affected}

resting breathing

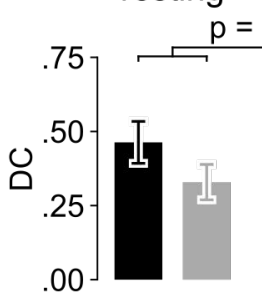

rostral $\rightarrow$ caudal
349

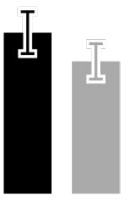

caudal $\rightarrow$ rostral
B

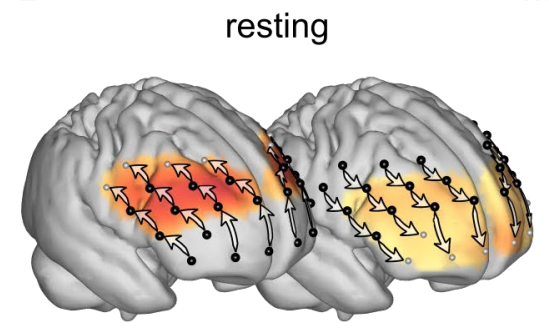

rostral $\rightarrow$ caudal caudal $\rightarrow$ rostral healthy

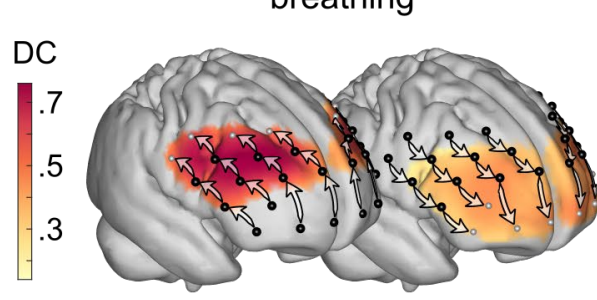

rostral $\rightarrow$ caudal caudal $\rightarrow$ rostral

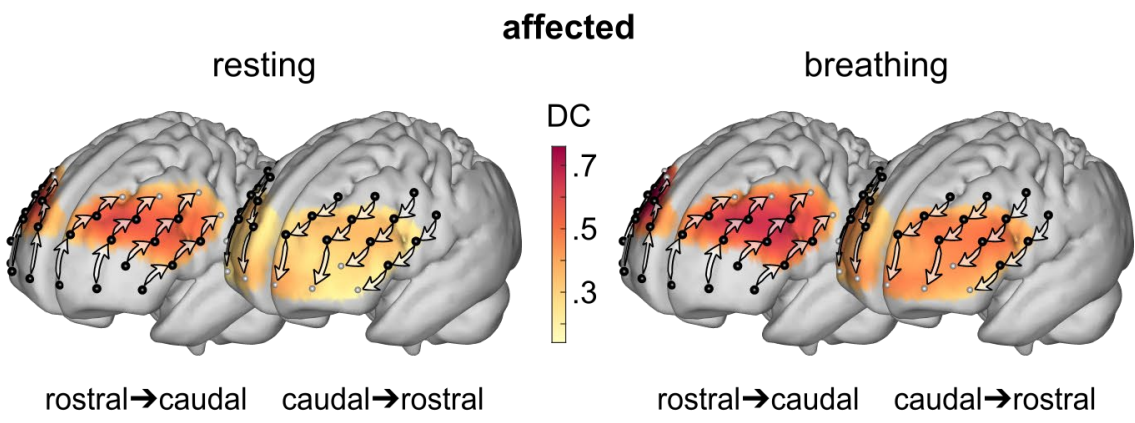

Figure 3: The rostro-caudal gradient in the healthy hemisphere is increased by metronomic breathing. A:

Metronomic breathing led to an overall increase of connectivity. Moreover, rostro-caudally directed influences in the healthy hemisphere increased over-proportionally during metronomic breathing (Model 1). As shown in Figure 4, this difference between conditions disappeared after intra-individually adjusting the estimation of directed connectivity for aBP oscillations, whereas the difference between the healthy and affected hemisphere was preserved. $\mathrm{N}=13$; bars represent least square means; error bars indicate $95 \%$ confidence intervals. B: Topographic illustration of the connectivity estimates, representing the influences from channels (black dots) toward caudally (left brain) and rostrally (right brain) neighboring channels as indicated by arrows; darker red colors signify stronger influences. Data for patients with stenosis of the right ICA were flipped such that the affected side is represented on the left hemisphere.

Adjusting estimates of directed connectivity for arterial blood pressure oscillations (intraindividual approach)

272 As metronomic breathing not only caused a marked increase in overall connectivity strength but also

273 changed the rostro-caudal gradient in the healthy hemisphere we sought to intra-individually control

274 for the potentially underlying mediation effect of aBP oscillations in a second analysis. To this end, we calculated the trivariate - i.e. partial - DC (PDC) between each fNIRS channel pair and the continuous aBP time series at the level of the individual subject, thereby removing estimated influences between brain regions that were mediated by aBP oscillations. The linear mixed model with the factors direction, hemisphere, and condition (as specified above) was fitted to these aBP-corrected 
connectivity estimates (Model 2) and demonstrated that changes induced by metronomic breathing were entirely mediated by aBP oscillations: Neither the main effect for condition $(\mathrm{p}=.377)$, nor any interaction involving condition was significant in the model fitted to the aBP-corrected data (all $\mathrm{p}>$ .354; see Table 1). However, main effects for direction $(\mathrm{F}(1,12)=19.8, \mathrm{p}=.0007)$ and hemisphere $(\mathrm{F}(1,1223)=4.0, \mathrm{p}=.044)$ as well as their interaction $(\mathrm{F}(1,1223)=14.5, \mathrm{p}=.0001)$ were significant.

284 Thus, intra-individually adjusting for effects of aBP oscillations not only allowed to correct breathing 285 induced artificial increases in the magnitudes of estimates of directed connectivity, but also revealed 286 that the connectivity gradient in the stenosed compared to the healthy hemisphere was generally attenuated irrespective of the condition (breathing vs. rest).

288 In order to explicitly test the effects of correcting the connectivity for aBP oscillations, supplementary 289 analyses directly compared the uncorrected DC estimates and the aBP-corrected PDC estimates 290 (Supplementary Model S6 and S7). These analyses confirmed that (i) the general over-estimation of 291 connectivity strength in both hemispheres and (ii) the increased rostro-caudal gradient in the healthy hemisphere caused by metronomic breathing disappeared after correcting for aBP oscillations. 
A healthy
resting breathing

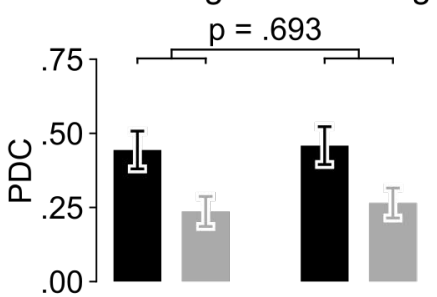

rostral $\rightarrow$ caudal caudal $\rightarrow$ rostral

\section{affected}

resting breathing

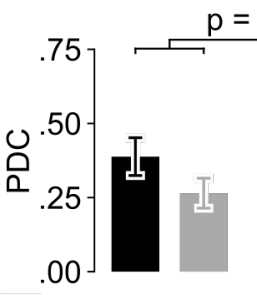

rostral $\rightarrow$ caudal
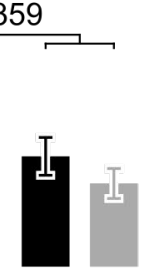

caudal $\rightarrow$ rostral
B

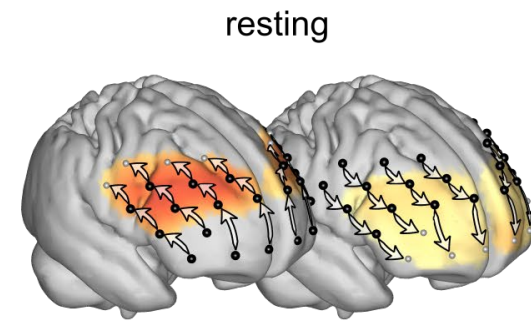

rostral $\rightarrow$ caudal caudal $\rightarrow$ rostral healthy

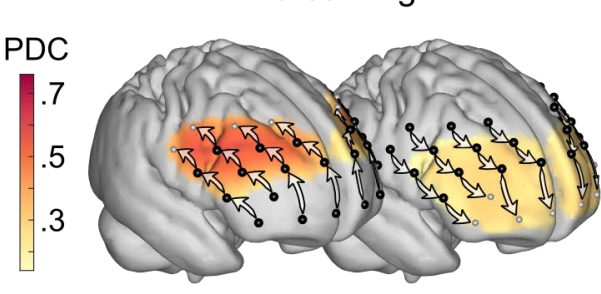

rostral $\rightarrow$ caudal caudal $\rightarrow$ rostral

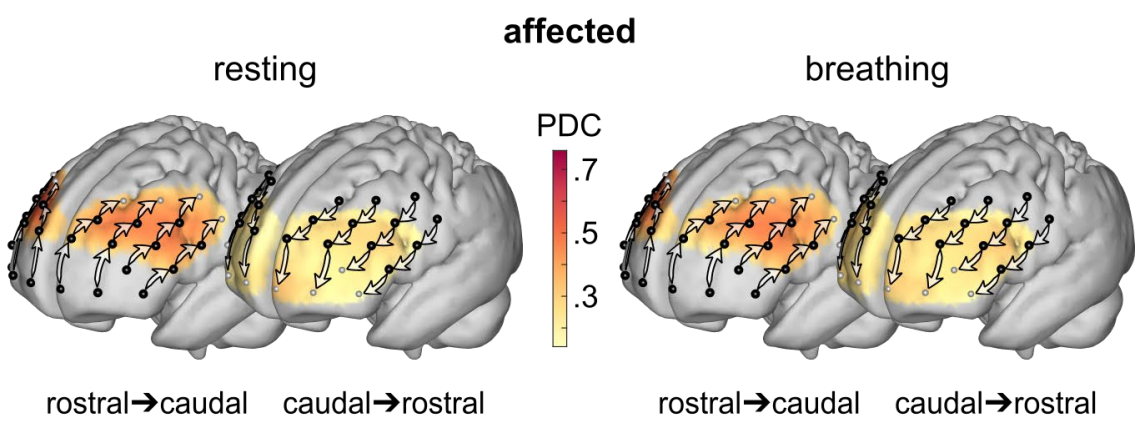

Fig 4: Including the aBP time series in the connectivity estimation entirely removed the effect of

295

296

297

298

299

300

301

302

303

304 metronomic breathing on the rostro-caudal connectivity. A: The effect that metronomic breathing exerted on the connectivity estimates was adjusted by including the aBP time series into the VAR model. This correction preserved the difference between hemispheres and revealed that the attenuation of the rostro-caudal gradient by ICA stenosis was independent of aBP fluctuations but reflected the compromised integrity of the functional network. P-values were obtained by fitting Model 1 (Figure 3) to the aBP-corrected connectivity estimates (Model 2). $\mathrm{N}=13$; bars represent least square means; error bars indicate $95 \%$ confidence intervals. B: Topographic illustration of the aBP-corrected connectivity estimates, representing the influences from channels (black dots) toward caudally (left brain) and rostrally (right brain) neighboring channels as indicated by arrows; darker red colors signify stronger influences. Data for patients with stenosis of the right ICA were flipped such that the affected side is represented on the left hemisphere.

Although the CBSI method used in the present analyses to improve signal quality is an established method that has been validated and compared to other artifact correction methods multiple times (e.g. Cooper et al. 2012; Brigadoi et al. 2014; Racz et al. 2017; Mukli et al. 2018; Fairclough et al. 2018; Fishburn et al. 2019), a systematic analysis of the impact of the CBSI method on Granger causality inference has not been performed yet. We therefore conducted control analyses on the uncorrected deoxygenated (dxyHb) and oxygenated (oxyHb) hemoglobin data (Supplementary Model S2-S5). The resulting connectivity estimates are depicted in Supplementary Figure S1 (DC) and S2 (PDC, corrected for influences by aBP fluctuations). In brief, the pattern of DC estimates, derived from the 
oxyHb data markedly deviated from those derived from the CBSI data, while the dxyHb-derived connectivity pattern was similar to the CBSI-derived pattern. Furthermore, the PDC estimates (i.e. the connectivity corrected for aBP influences) were similar for the oxyHb, dxyHb, and CBSI data suggesting that the deviating results for the oxyHb-derived DC estimates were due to the higher susceptibility of the oxyHb measurement to physiological noise as previously reported (Obrig et al. 2000; Zhang et al. 2009; Kirilina et al. 2012; Sutoko et al. 2019). This again corroborates our finding that including the aBP signal in PDC estimation effectively controlled for bias induced by physiological noise.

\section{Low-frequency aBP variance is reflected in low-frequency fNIRS variance}

The strong intra-individual effect of including peripheral measured aBP oscillations in the PDC estimation of the directed functional connectivity raises the question whether the magnitude of the aBP oscillations is reflected in the low-frequency component of the fNIRS signal. Correlations between the peak power spectral density (PSD, in $\mathrm{dB}$ ) of the peripheral aBP and the fNIRS signals in the frequency band between .06 and $.12 \mathrm{~Hz}$ (Fig. 5a) revealed strong associations between the low-frequency variance of the aBP and the fNIRS signals across patients (Fig. 5b). The spatial distribution of correlation coefficients (Fig. 6b) indicated considerable variation across hemispheres and conditions. A $2 \times 2$ repeated measures analysis of variance (ANOVA; performed using the ez package for $\mathrm{R}$, version 4.4-0; Lawrence, 2016) on the fisher-transformed Pearson correlation coefficients (Model 3, calculated with the homologous channels as the unit of observation) showed that correlations were stronger in the affected than in the healthy hemisphere $\left(F(1,15)=11.63, p=.004\right.$, generalized $\eta^{2}=$ .194; Fig. 6a). Furthermore, the significant interaction effect between hemisphere and condition $\left(\mathrm{F}(1,15)=13.40, \mathrm{p}=.002\right.$, generalized $\left.\eta^{2}=.043\right)$ revealed that the correlation difference between conditions was mainly driven by elevated PSD correlations in the healthy hemisphere during metronomic breathing compared to resting state (mean difference: .122, $\mathrm{t}(15)=1.72, \mathrm{p}=.11$ ); the difference between conditions in the affected hemisphere was considerably smaller (mean difference: $.035, \mathrm{t}(15)=.568, \mathrm{p}=.58)$. These effects clearly demonstrate the impairment of the vasomotor reactivity in the stenosed hemisphere (where aBP fluctuations appeared almost undamped), not only during metronomic breathing but also during rest. Averaged across channels and conditions, the low- 
341 frequency PSD of the fNIRS signal shared $23 \%$ of variance with the low-frequency PSD of the 342 peripheral aBP (Fig. 5b).

A
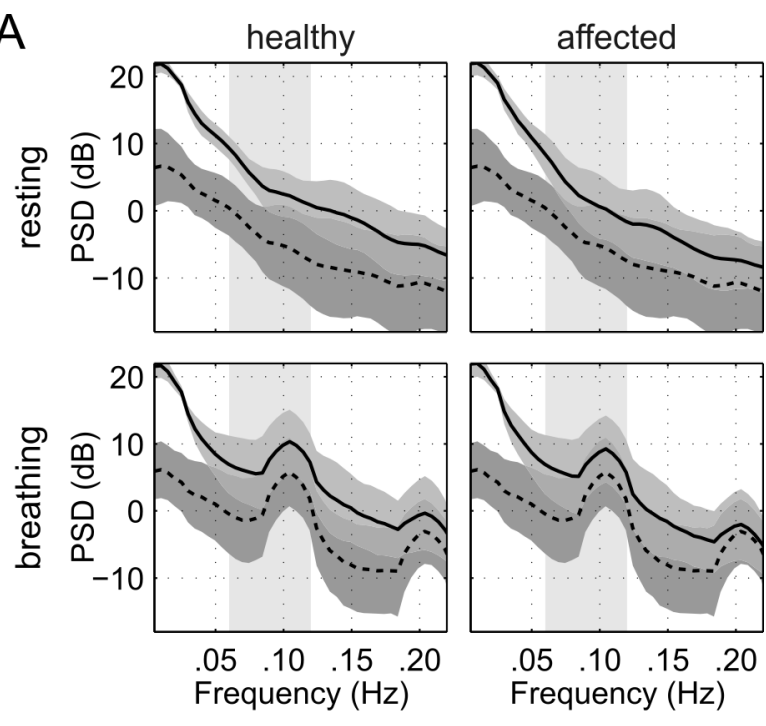

- fNIRS

(....- aBP

$\mathrm{B}$

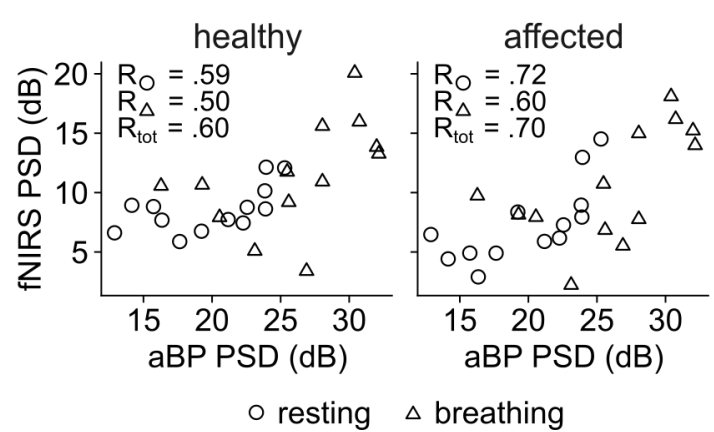

344 Fig. 5: The power spectral density (PSD) of the fNIRS signal and the continuously measured aBP. A: PSD of the fNIRS (solid line) and the aBP (dashed line) signals during resting state (top panels) and metronomic breathing (bottom panels) separately for the healthy and the stenosed hemisphere. The aBP was measured by a finger plethysmograph and its PSD is shown twice along with the fNIRS PSD for both hemispheres to facilitate comparison. The fNIRS PSDs were averaged across channels within each hemisphere; lines represent averages across patients and gray patches indicate standard deviations across patients. The area shaded in light gray marks the frequency band between .06 and $.12 \mathrm{~Hz}$ which was used for analyses. B: Scatter plot of aBP and fNIRS PSDs. From each PSD spectrum the maximum in the frequency band of interest was used. The fNIRS PSD values were averaged across channels included in the mixed model analyses. The correlations between PSDs for single fNIRS channels are shown topographically in Figure $6 \mathrm{~b}$. 

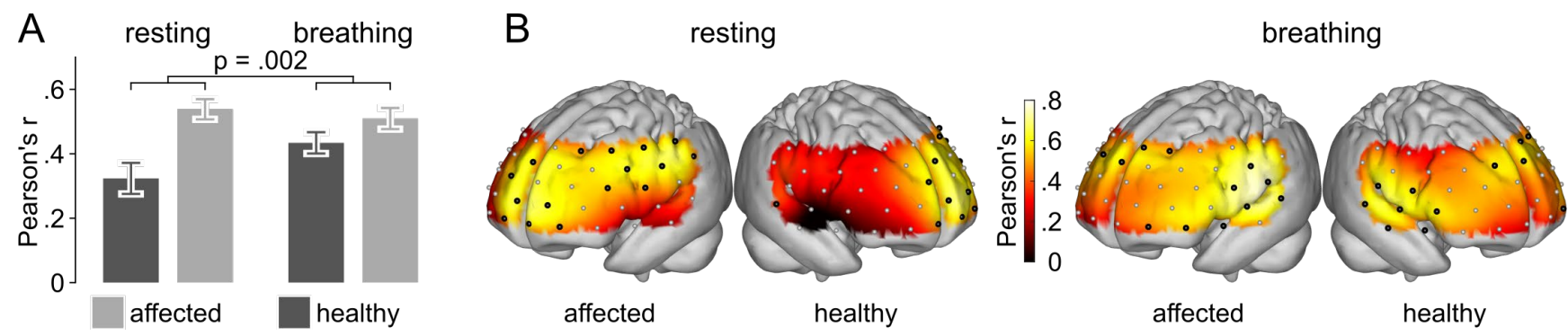

Fig 6: The coupling between low-frequency aBP and fNIRS signal variance in the healthy hemisphere is increased by metronomic breathing. A: The correlations between the peak PSD in the frequency band [.06 .12] $\mathrm{Hz}$ of the aBP and each fNIRS channel were calculated, Fisher-Z-transformed, and analyzed in a repeated measures ANOVA (Model 3). In the resting state the impairment of the vasomotor reactivity by the ICA stenosis caused high correlations between aBP and fNIRS PSDs in the affected hemisphere, which were not further increased by metronomic breathing. In contrast, the vasculature of the healthy hemisphere with its intact autoregulation minimized PSD correlations in the resting state. However, metronomic breathing induced strong aBP oscillations also in the healthy hemisphere which lead to a marked increase in PSD correlations. Taken together, coupling between aBP and fNIRS signal variance increased by metronomic breathing in the healthy but not in the stenosed hemisphere. $\mathrm{N}=13$; bars represent mean correlation coefficients; error bars indicate standard error of the mean. B: Topographic illustration of correlation coefficients. Black dots mark channels for which correlation was significant ( $\mathrm{p}<.05$, uncorrected). For the sake of completeness, this figure shows correlations for all fNIRS channels, i.e. also for those not included in the connectivity analyses, while the ANOVA considered only channels that contributed to at least one connection (cf. Fig. 1). Data for patients with stenosis of the right ICA were flipped such that the affected side is represented on the left hemisphere.

\section{Low-frequency fNIRS-variance accounts for the effect of metronomic breathing (inter-} individual approach)

372 Given the strong association between the low-frequency variation in the aBP and the fNIRS signals across patients, we further asked whether the fNIRS PSD can serve as a proxy for the intra-individual aBP fluctuations in order to explain the effect of breathing on estimates of directed connectivity on the inter-individual level. As this could provide a general possibility to correct the connectivity estimates for the aBP induced bias without relying on monitoring the peripheral aBP time course, we tested whether the low-frequency fNIRS PSD can account for aBP-induced variance in the connectivity estimates. Accordingly, the first model (using the uncorrected connectivity estimates) was extended by the peak PSD of the fNIRS signals in the frequency band between .06 and $.12 \mathrm{~Hz}$ as a covariate, including all resulting 2-, 3- and 4-way interactions with the nominal predictors (Model 4). As there is 
one peak PSD value for each fNIRS channel, we used the mean value of the respective channel pairs

382

for the corresponding connections. In addition to main effects for direction $(\mathrm{F}(1,28.0)=4.5, \mathrm{p}=.042)$ and condition $(\mathrm{F}(1,1028)=6.2, \mathrm{p}=.013)$, this model revealed a simple effect of the continuous predictor low-frequency PSD $(\mathrm{F}(1,224.8)=6.2, \mathrm{p}=.014)$. As expected, the 2-way interaction between direction and hemisphere $(\mathrm{F}(1,1207.1)=1.4, \mathrm{p}=.234)$ and, more importantly, the 3-way interaction between direction, hemisphere, and condition $(\mathrm{F}(1,1205.9)=.9, \mathrm{p}=.340)$ disappeared. Instead, the significant 3-way interaction between direction, hemisphere, and low-frequency PSD $(\mathrm{F}(1,1207.3)=$ $7.8, \mathrm{p}=.005)$ revealed a positive correlation between the rostro-caudal gradient and the variance in the low-frequency fNIRS signal component in the healthy hemisphere only (Fig. 7, left panel; contrast between slopes for low-frequency PSD of rostrally and caudally directed influences in the healthy hemisphere: $\mathrm{p}=.008)$. In the affected hemisphere, low-frequency PSD did not predict the difference between rostrally and caudally directed influences $(p=.239)$. Thus, the low-frequency variance of the fNIRS signal (i) moderated the effect of hemisphere (i.e. of the ICA stenosis) on the rostro-caudal gradient and (ii) mediated the effect of condition (i.e. of aBP oscillations) on the gradient in the healthy hemisphere. No further effects were significant (all $p>.198$ ).

F7

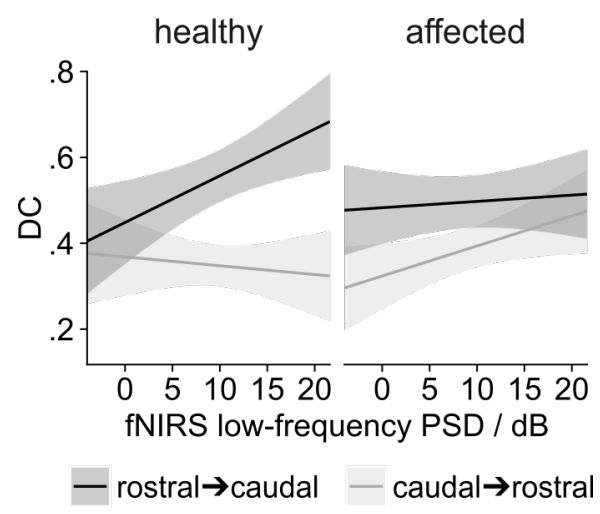

Fig 7: The fNIRS signal power explained variance in the connectivity estimates in the healthy hemisphere. rostro-caudal gradient, i.e. the effect of condition (Fig. 3) disappeared after including the fNIRS PSD as a predictor in the linear mixed model (Model 4). $\mathrm{N}=13$; model predictions are shown with $95 \%$ non-simultaneous confidence bands. 
403 Table 1: Type III statistics for second-level models

\begin{tabular}{|c|c|c|c|c|c|}
\hline Model (Figures) & Effect & df & Error df & F value & p value \\
\hline \multirow{7}{*}{$\begin{array}{l}\text { Model 1 } \\
\text { DC LMM } \\
\text { (Figure 3) }\end{array}$} & direction & 1 & 12 & 14.57 & .0025 \\
\hline & condition & 1 & 1212 & 60.97 & $1 \times 10^{-14}$ \\
\hline & hemisphere & 1 & 1212 & .22 & .6419 \\
\hline & direction $\times$ condition & 1 & 1212 & .63 & .4292 \\
\hline & direction $\times$ hemisphere & 1 & 1212 & 12.20 & .0005 \\
\hline & condition $\times$ hemisphere & 1 & 1212 & .64 & .4250 \\
\hline & direction $\times$ condition $\times$ hemisphere & 1 & 1212 & 4.48 & .0345 \\
\hline \multirow{7}{*}{$\begin{array}{l}\text { Model } 2 \\
\text { PDC LMM } \\
\text { (aBP corrected } \\
\text { connectivity; } \\
\text { Figure 4) }\end{array}$} & direction & 1 & 12 & 19.79 & .0008 \\
\hline & condition & 1 & 1223 & .78 & .3767 \\
\hline & hemisphere & 1 & 1223 & 4.05 & .0444 \\
\hline & direction $\times$ condition & 1 & 1223 & .86 & .3537 \\
\hline & direction $\times$ hemisphere & 1 & 1223 & 14.49 & .0001 \\
\hline & condition $\times$ hemisphere & 1 & 1223 & .79 & .3741 \\
\hline & direction $\times$ condition $\times$ hemisphere & 1 & 1223 & .14 & .7117 \\
\hline \multirow{3}{*}{$\begin{array}{l}\text { Model } 3 \\
\text { ANOVA of PSD } \\
\text { correlations (Figure 6) }\end{array}$} & condition & 1 & 15 & .47 & .5027 \\
\hline & hemisphere & 1 & 15 & 11.63 & .0039 \\
\hline & condition $\times$ hemisphere & 1 & 15 & 13.40 & .0023 \\
\hline \multirow{15}{*}{$\begin{array}{l}\text { Model } 4 \\
\text { DC LMM with fNIRS } \\
\text { PSD covariate } \\
\text { (Figure 7) }\end{array}$} & $\operatorname{dir}$ & 1 & 28 & 4.54 & .0419 \\
\hline & condition & 1 & 1028 & 6.17 & .0132 \\
\hline & hemisphere & 1 & 1230 & .00 & .9591 \\
\hline & $\mathrm{PSD}_{\mathrm{fNIRS}}$ & 1 & 225 & 6.16 & .0138 \\
\hline & direction $\times$ condition & 1 & 1206 & .21 & .6451 \\
\hline & direction $\times$ hemisphere & 1 & 1207 & 1.41 & .2345 \\
\hline & condition $\times$ hemisphere & 1 & 1225 & 1.40 & .2369 \\
\hline & direction $\times$ PSD $_{\mathrm{fNIRS}}$ & 1 & 1211 & 1.66 & .1982 \\
\hline & condition $\times \mathrm{PSD}_{\mathrm{fNIRS}}$ & 1 & 1040 & .05 & .8242 \\
\hline & hemisphere $\times \mathrm{PSD}_{\mathrm{fNIRS}}$ & 1 & 1233 & .06 & .8047 \\
\hline & direction $\times$ condition $\times$ hemisphere & 1 & 1206 & .91 & .3402 \\
\hline & direction $\times$ condition $\times \mathrm{PSD}_{\mathrm{fNIRS}}$ & 1 & 1207 & .47 & .4933 \\
\hline & direction $\times$ hemisphere $\times$ PSD $_{\mathrm{fNIRS}}$ & 1 & 1207 & 7.83 & .0052 \\
\hline & condition $\times$ hemisphere $\times$ PSD $_{\mathrm{fNIRS}}$ & 1 & 1228 & .86 & .3544 \\
\hline & direction $\times$ condition $\times$ hemisphere $\times$ PSD $_{\mathrm{fNIRS}}$ & 1 & 1206 & .32 & .5746 \\
\hline
\end{tabular}
with Satterthwaite approximation of degrees of freedom. Abbreviations: ANOVA, analysis of variance; DC, directed coherence; df, degrees of freedom; LMM, linear mixed model; PDC, partial directed coherence; PSD, power spectral density (in the low frequency band).

\section{Discussion}

The present study addressed effects of physiological noise and vasomotor reactivity on Granger-causal cross-spectral analyses of multi-channel fNIRS data with high temporal resolution. Specifically, we 
asked (i) whether physiological noise, which inevitably contaminates fNIRS measurements, biases

412 Granger-causality estimates of directed connectivity along the rostro-caudal axis in the PFC, and (ii)

413 whether severe carotid artery stenosis, which impairs the neurovascular coupling in the PFC (Rossini

414 et al. 2004; Bokkers et al. 2010; Novak and Hajjar 2010; Hartkamp et al. 2012; Novak 2012) also

415 affects the rostro-caudal hierarchical organization of the PFC. We found that the impact of strong aBP

416 oscillations on the directed connectivity estimates was direction-unspecific and could be removed by

417 including the aBP time course in the connectivity estimation. Additionally, the effect of aBP

418 oscillations on the directed functional connectivity was explained by the low-frequency power of the

419 fNIRS signal. Furthermore, results showed that the rostro-caudal functional organization of the PFC in

420 the affected hemisphere was specifically attenuated by ICA stenosis. Taken together, Granger-causal

421 cross-spectral analyses of resting-state fNIRS measurements were robust against ordinary levels of physiological noise but sensitive to the integrity of the neurovascular system.

The enhancing effect of the breathing-induced fNIRS signal oscillations on the connectivity estimates demonstrated that the manipulation of this physiological parameter biased the estimation of directed connectivity. As it is very unlikely that this change in connectivity reflects a change in functional organization evoked by the instruction of metronomic breathing, the increased low-frequency signal variance (at .1 Hz) probably caused elevated estimates of functional connections. This interpretation is supported by three observations: (i) The increase in connectivity during metronomic breathing was more pronounced in the healthy compared to the stenosed hemisphere. As ICA stenosis caused a reduction of the vasomotor reactivity and impaired the cerebral autoregulation in the affected hemisphere (Bokkers et al. 2010; Reinhard et al. 2014), the vasculature had less capacity to compensate aBP fluctuations, presumably already in the resting state. Thus, the coupling between aBP and the fNIRS signal was increased by metronomic breathing only in the healthy but not in the stenosed hemisphere (Fig. 6) and, accordingly, exerted a stronger effect on estimates of directed connectivity in the healthy than in the stenosed hemisphere. (ii) The effect of breathing-induced increases of directed connectivity completely disappeared after including the aBP signal (the continuous finger photoplethysmogram measurements) in the intra-individual connectivity estimation. 
systemic aBP fluctuations (i.e. non-neural signal variance). (iii) Besides the peripheral, global hemodynamic signal, the local, low-frequency power spectral density of the fNIRS time series also reflected the effect of metronomic breathing on the connectivity, i.e. the low-frequency power spectral density accounted for the difference between conditions. The fact that the connectivity gradient was predicted by the low-frequency signal variance only in the healthy and not in the affected hemisphere indicates that strong vasomotion can bias the Granger-causal estimates of directed connectivity. Taken together, we conclude that the impact of peripheral physiological parameters, notably aBP oscillations, on Granger-causality inference can be adjusted by taking inter-individual differences in fNIRS signal variance into account. However, when comparing connectivity estimated from measurements under conditions with considerable variations in aBP, the present results advise to include the aBP time series in the intra-individual connectivity estimation in order to preclude that differences in connectivity are caused simply by autoregulatory vasomotion. In this respect, it is worth noting that peripheral photoplethysmography used to measure slow aBP fluctuations in the present study also capture cardiac and respiratory pulsations, which have been shown to constitute noise sources in functional hemodynamic measurements as well (Frederick et al. 2012). Thus, improvement of connectivity estimates by including the aBP time-series in the VAR model may have also relied on the suppression of other systemic physiological noise sources.

In contrast to the effect of metronomic breathing, the effect of ICA stenosis on the connectivity estimates was persistent after correcting for the global hemodynamic component as well as after taking the local signal variance into account. The difference in connectivity between healthy and affected hemisphere therefore reflected the integrity of the functional network organization rather than mere physiological processes. This finding was highly expected as ICA stenosis has been shown before not only to impair the hemodynamic response (Rossini et al. 2004) and functional connectivity (Avirame et al. 2015) but also to cause functional deficits like cognitive decline (Novak and Hajjar 2010; Novak 2012). However, as no behavioral data were available for the present sample of patients, the relationship between alterations in the rostro-caudal connectivity gradient and specific cognitive function requires further research. Moreover, future studies should take advantage of more recent advancements in fNIRS technology and, specifically, capitalize on multi-distance and tomographic 
467

measurements to more efficiently eliminate extra-cerebral signal components (Habermehl et al. 2012; Eggebrecht et al. 2014; Gagnon et al. 2014; Sato et al. 2016).

In summary, the high temporal resolution of fNIRS renders Granger-causality analyses of hemodynamic measurements possible and allows the comparison of different conditions provided that physiological parameters like aBP are controlled. In line with previous studies we demonstrated that (i) peripheral measurements of systemic hemodynamic processes can be used to correct functional connectivity estimates for physiological noise (Frederick et al. 2012; Tong et al. 2013; Sutoko et al. 2019) and that (ii) ICA stenosis impairs functional network organization (Avirame et al. 2015).

\section{References}

Avirame K, Lesemann A, List J, et al (2015) Cerebral autoregulation and brain networks in occlusive processes of the internal carotid artery. J Cereb Blood Flow Metab 35:240-247. doi: $10.1038 / \mathrm{jcbfm} .2014 .190$

Badre D, D’Esposito M (2007) Functional magnetic resonance imaging evidence for a hierarchical organization of the prefrontal cortex. J Cogn Neurosci 19:2082-2099

Badre D, Nee DE (2018) Frontal Cortex and the Hierarchical Control of Behavior. Trends Cogn Sci 22:170-188. doi: 10.1016/j.tics.2017.11.005

Barnett L, Seth AK (2017) Detectability of Granger causality for subsampled continuous-time neurophysiological processes. J Neurosci Methods 275:93-121. doi: 10.1016/j.jneumeth.2016.10.016

Barnett L, Seth AK (2011) Behaviour of Granger causality under filtering: theoretical invariance and practical application. J Neurosci Methods 201:404-19. doi: 10.1016/j.jneumeth.2011.08.010

Bates D, Mächler M, Bolker B, Walker S (2015) Fitting Linear Mixed-Effects Models Using lme4. J Stat Softw 67:1-48. doi: 10.18637/jss.v067.i01

Biswal B, Yetkin FZ, Haughton VM, Hyde JS (1995) Functional connectivity in the motor cortex of 

resting human brain using echo-planar MRI. Magn Reson Med 34:537-41. doi: 10.1002/mrm. 1910340409

493

494

Blumenfeld RS, Nomura EM, Gratton C, D'Esposito M (2013) Lateral prefrontal cortex is organized into parallel dorsal and ventral streams along the rostro-caudal axis. Cereb Cortex 23:2457-66. doi: $10.1093 /$ cercor/bhs223

Bokkers RPH, van Osch MJP, van der Worp HB, et al (2010) Symptomatic carotid artery stenosis: impairment of cerebral autoregulation measured at the brain tissue level with arterial spinlabeling MR imaging. Radiology 256:201-8. doi: 10.1148/radiol.10091262

Brigadoi S, Ceccherini L, Cutini S, et al (2014) Motion artifacts in functional near-infrared spectroscopy: A comparison of motion correction techniques applied to real cognitive data. Neuroimage 85:181-191. doi: 10.1016/j.neuroimage.2013.04.082

Brigadoi S, Cooper RJ (2015) How short is short? Optimum source-detector distance for shortseparation channels in functional near-infrared spectroscopy. Neurophotonics 2:025005. doi: 10.1117/1.NPh.2.2.025005

Christoff K, Gabrieli JDE (2000) The frontopolar cortex and human cognition: Evidence for a rostrocaudal hierarchical organization within the human prefrontal cortex. Psychobiology 28:168-186

Cooper RJ, Selb J, Gagnon L, et al (2012) A Systematic Comparison of Motion Artifact Correction Techniques for Functional Near-Infrared Spectroscopy. Front Neurosci 6:1-10. doi: $10.3389 /$ fnins. 2012.00147

Cui X, Bray S, Reiss AL (2010) Functional near infrared spectroscopy (NIRS) signal improvement based on negative correlation between oxygenated and deoxygenated hemoglobin dynamics. Neuroimage 49:3039-46. doi: 10.1016/j.neuroimage.2009.11.050

de Bray JM, Glatt B (1995) Quantification of Atheromatous Stenosis in the Extracranial Internal Carotid Artery. Cerebrovasc Dis 5:414-426. doi: 10.1159/000107895 
Delpy DT, Cope M, van der Zee P, et al (1988) Estimation of optical pathlength through tissue from direct time of flight measurement. Phys Med Biol 33:1433-42

Deshpande G, Hu X (2012) Investigating effective brain connectivity from fMRI data: past findings and current issues with reference to Granger causality analysis. Brain Connect 2:235-245. doi: 10.1089/brain.2012.0091 [doi]

Deshpande G, Sathian K, Hu X (2010) Effect of hemodynamic variability on Granger causality analysis of fMRI. Neuroimage 52:884-896. doi: 10.1016/j.neuroimage.2009.11.060

Eggebrecht AT, Ferradal SL, Robichaux-Viehoever A, et al (2014) Mapping distributed brain function and networks with diffuse optical tomography. Nat Photonics 8:448-454. doi: 10.1038/nphoton.2014.107

Fairclough SH, Burns C, Kreplin U (2018) FNIRS activity in the prefrontal cortex and motivational intensity: impact of working memory load, financial reward, and correlation-based signal improvement. Neurophotonics 5:035001. doi: 10.1117/1.NPh.5.3.035001

Fishburn FA, Ludlum RS, Vaidya CJ, Medvedev A V. (2019) Temporal Derivative Distribution Repair (TDDR): A motion correction method for fNIRS. Neuroimage 184:171-179. doi: 10.1016/j.neuroimage.2018.09.025

Florin E, Gross J, Pfeifer J, et al (2010) The effect of filtering on Granger causality based multivariate causality measures. Neuroimage $50: 577-588$. doi: http://dx.doi.org/10.1016/j.neuroimage.2009.12.050

Frederick B deB, Nickerson LD, Tong Y (2012) Physiological denoising of BOLD fMRI data using Regressor Interpolation at Progressive Time Delays (RIPTiDe) processing of concurrent fMRI and near-infrared spectroscopy (NIRS). Neuroimage 60:1913-23. doi: 10.1016/j.neuroimage.2012.01.140

Friston K, Moran R, Seth AK (2013) Analysing connectivity with Granger causality and dynamic causal modelling. Curr Opin Neurobiol 23:172-8. doi: 10.1016/j.conb.2012.11.010 
Friston KJ, Bastos AM, Oswal A, et al (2014) Granger causality revisited. Neuroimage 101:796-808. doi: 10.1016/j.neuroimage.2014.06.062

Fuster JM (2008) The Prefrontal Cortex, 4th edn. Academic Press/Elsevier, London

Gagnon L, Yücel M a., Boas D a., Cooper RJ (2014) Further improvement in reducing superficial contamination in NIRS using double short separation measurements. Neuroimage 85:127-135. doi: 10.1016/j.neuroimage.2013.01.073

Germon TJ, Evans PD, Barnett NJ, et al (1999) Cerebral near infrared spectroscopy: emitter-detector separation must be increased. Br J Anaesth 82:831-837

Granger CWJ (1969) Investigating Causal Relations by Econometric Models and Cross-spectral Methods. Econometrica 37:424. doi: 10.2307/1912791

Habermehl C, Holtze S, Steinbrink J, et al (2012) Somatosensory activation of two fingers can be discriminated with ultrahigh-density diffuse optical tomography. Neuroimage 59:3201-11. doi: 10.1016/j.neuroimage.2011.11.062

Hartkamp NS, Hendrikse J, van der Worp HB, et al (2012) Time Course of Vascular Reactivity Using Repeated Phase-Contrast MR Angiography in Patients With Carotid Artery Stenosis. Stroke 43:553-556. doi: 10.1161/STROKEAHA.111.637314

Julien C (2006) The enigma of Mayer waves: Facts and models. Cardiovasc Res 70:12-21. doi: 10.1016/j.cardiores.2005.11.008

Kirilina E, Jelzow A, Heine A, et al (2012) The physiological origin of task-evoked systemic artefacts in functional near infrared spectroscopy. Neuroimage 61:70-81. doi: 10.1016/j.neuroimage.2012.02.074

Koechlin E, Ody C, Kouneiher F (2003) The architecture of cognitive control in the human prefrontal cortex. Science 302:1181-5. doi: 10.1126/science.1088545

564 Kuznetsova A, Bruun Brockhoff P, Haubo Bojesen Christensen R (2016) lmerTest: Tests in Linear 

Mixed Effects Models

Lenth R V (2016) Least-Squares Means: The R Package 1smeans. J Stat Softw 69:1-33. doi: 10.18637/jss.v069.i01

Mader W, Feess D, Lange R, et al (2008) On the Detection of Direct Directed Information Flow in fMRI. IEEE J Sel Top Signal Process 2:965-974. doi: 10.1109/JSTSP.2008.2008260

Margulies DS, Ghosh SS, Goulas A, et al (2016) Situating the default-mode network along a principal gradient of macroscale cortical organization. Proc Natl Acad Sci U S A 113:12574-12579. doi: $10.1073 /$ pnas. 1608282113

Mukli P, Nagy Z, Racz FS, Eke HP (2018) Impact of Healthy Aging on Multifractal Hemodynamic Fluctuations in the Human Prefrontal Cortex. Front Physiol 9:1072. doi: 10.3389/fphys.2018.01072

Nee DE, D’Esposito M (2016) The hierarchical organization of the lateral prefrontal cortex. Elife 5:126. doi: $10.7554 /$ eLife. 12112

Noordmans HJ, van Blooijs D, Siero JCW, et al (2018) Detailed view on slow sinusoidal, hemodynamic oscillations on the human brain cortex by Fourier transforming oxy/deoxy hyperspectral images. Hum Brain Mapp 39:3558-3573. doi: 10.1002/hbm.24194

Novak V (2012) Cognition and Hemodynamics. Curr Cardiovasc Risk Rep 6:380-396. doi: $10.1007 / \mathrm{s} 12170-012-0260-2$

Novak V, Hajjar I (2010) The relationship between blood pressure and cognitive function. Nat Rev Cardiol 7:686-98. doi: 10.1038/nrcardio.2010.161

Obrig H, Neufang M, Wenzel R, et al (2000) Spontaneous low frequency oscillations of cerebral hemodynamics and metabolism in human adults. Neuroimage 12:623-39. doi: 10.1006/nimg.2000.0657 
Okada E, Firbank M, Schweiger M, et al (1997) Theoretical and experimental investigation of nearinfrared light propagation in a model of the adult head. Appl Opt 36:21-31

Pfurtscheller G, Schwerdtfeger A, Brunner C, et al (2017) Distinction between Neural and Vascular BOLD Oscillations and Intertwined Heart Rate Oscillations at $0.1 \mathrm{~Hz}$ in the Resting State and during Movement. PLoS One 12:e168097. doi: 10.1371/journal.pone.0168097

Racz FS, Mukli P, Nagy Z, Eke A (2017) Increased prefrontal cortex connectivity during cognitive challenge assessed by fNIRS imaging. Biomed Opt Express 8:3842-3855. doi: 10.1364/BOE.8.003842

Reinhard M, Müller T, Guschlbauer B, et al (2003a) Dynamic cerebral autoregulation and collateral flow patterns in patients with severe carotid stenosis or occlusion. Ultrasound Med Biol 29:1105-1113. doi: 10.1016/S0301-5629(03)00954-2

Reinhard M, Roth M, Müller T, et al (2003b) Cerebral autoregulation in carotid artery occlusive disease assessed from spontaneous blood pressure fluctuations by the correlation coefficient index. Stroke 34:2138-44. doi: 10.1161/01.STR.0000087788.65566.AC

Reinhard M, Schumacher FK, Rutsch S, et al (2014) Spatial mapping of dynamic cerebral autoregulation by multichannel near-infrared spectroscopy in high-grade carotid artery disease. $\mathrm{J}$ Biomed Opt 19:097005. doi: 10.1117/1.JBO.19.9.097005

Roebroeck A, Formisano E, Goebel R (2005) Mapping directed influence over the brain using Granger causality and fMRI. Neuroimage 25:230-42. doi: 10.1016/j.neuroimage.2004.11.017

Rossini PM, Altamura C, Ferretti A, et al (2004) Does cerebrovascular disease affect the coupling between neuronal activity and local haemodynamics? Brain 127:99-110. doi: 10.1093/brain/awh012

Santosa H, Aarabi A, Perlman SB, Huppert TJ (2017) Characterization and correction of the falsediscovery rates in resting state connectivity using functional near-infrared spectroscopy. J Biomed Opt 22:55002. doi: 10.1117/1.JBO.22.5.055002 
Sato T, Nambu I, Takeda K, et al (2016) Reduction of global interference of scalp-hemodynamics in functional near-infrared spectroscopy using short distance probes. Neuroimage 141:120-132. doi: 10.1016/j.neuroimage.2016.06.054

Satterthwaite TD, Wolf DH, Loughead J, et al (2012) Impact of in-scanner head motion on multiple measures of functional connectivity: relevance for studies of neurodevelopment in youth. Neuroimage 60:623-32. doi: 10.1016/j.neuroimage.2011.12.063

Schelter B, Winterhalder M, Eichler M, et al (2006) Testing for directed influences among neural signals using partial directed coherence. J Neurosci Methods 152:210-9. doi: 10.1016/j.jneumeth.2005.09.001

Schippers MB, Renken R, Keysers C (2011) The effect of intra- and inter-subject variability of hemodynamic responses on group level Granger causality analyses. Neuroimage 57:22-36. doi: 10.1016/j.neuroimage.2011.02.008

Scholkmann F, Kleiser S, Metz AJ, et al (2014) A review on continuous wave functional near-infrared spectroscopy and imaging instrumentation and methodology. Neuroimage 85 Pt 1:6-27. doi: 10.1016/j.neuroimage.2013.05.004

Schumacher FK, Schumacher LV, Schelter BO, Kaller CP (2019) Functionally dissociating ventrodorsal components within the rostro-caudal hierarchical organization of the human prefrontal cortex. Neuroimage 185:398-407. doi: 10.1016/j.neuroimage.2018.10.048

Scouten A, Papademetris X, Constable RT (2006) Spatial resolution, signal-to-noise ratio, and smoothing in multi-subject functional MRI studies. Neuroimage 30:787-793. doi: 10.1016/j.neuroimage.2005.10.022

Smith SM, Bandettini PA, Miller KL, et al (2012) The danger of systematic bias in group-level FMRIlag-based causality estimation. Neuroimage 59:1228-1229. doi: 10.1016/j.neuroimage.2011.08.015

Stokes PA, Purdon PL (2017) A study of problems encountered in Granger causality analysis from a 

neuroscience perspective. Proc Natl Acad Sci 114:E7063-E7072. doi: 10.1073/pnas.1704663114

640

641

642

643

644

645

646

647

648

649

650

651

652

653

654

655

656

657

658

659

660

661

Sutoko S, Chan YL, Obata A, et al (2019) Denoising of neuronal signal from mixed systemic lowfrequency oscillation using peripheral measurement as noise regressor in near-infrared imaging. Neurophotonics 6:015001. doi: 10.1117/1.NPh.6.1.015001

Takahashi T, Takikawa Y, Kawagoe R, et al (2011) Influence of skin blood flow on near-infrared spectroscopy signals measured on the forehead during a verbal fluency task. Neuroimage 57:991-1002. doi: 10.1016/j.neuroimage.2011.05.012

Tong Y, Frederick BD (2010) Time lag dependent multimodal processing of concurrent fMRI and near-infrared spectroscopy (NIRS) data suggests a global circulatory origin for low-frequency oscillation signals in human brain. Neuroimage 53:553-64. doi: 10.1016/j.neuroimage.2010.06.049

Tong Y, Hocke LM, Licata SC, Frederick B deB (2012) Low-frequency oscillations measured in the periphery with near-infrared spectroscopy are strongly correlated with blood oxygen leveldependent functional magnetic resonance imaging signals. J Biomed Opt 17:106004. doi: 10.1117/1.JBO.17.10.106004

Tong Y, Hocke LM, Nickerson LD, et al (2013) Evaluating the effects of systemic low frequency oscillations measured in the periphery on the independent component analysis results of resting state networks. Neuroimage 76:202-215. doi: 10.1016/j.neuroimage.2013.03.019

Webb JT, Ferguson M a, Nielsen J a, Anderson JS (2013) BOLD Granger causality reflects vascular anatomy. PLoS One 8:e84279. doi: 10.1371/journal.pone.0084279

Winder AT, Echagarruga C, Zhang Q, Drew PJ (2017) Weak correlations between hemodynamic signals and ongoing neural activity during the resting state. Nat Neurosci 20:1761-1769. doi: 10.1038/s41593-017-0007-y

Zhang Q, Strangman GE, Ganis G (2009) Adaptive filtering to reduce global interference in noninvasive NIRS measures of brain activation: How well and when does it work? Neuroimage 
